A N N A L E S Annales de Bretagne et des Pays de l'Ouest

Le journal d'un chanoine du Mans. Nepveu de La Manouillère (1759-1807)

\title{
Olivier Charles
}

\section{(2) OpenEdition}

1 Journals

Édition électronique

URL : http://journals.openedition.org/abpo/2802

DOI : $10.4000 /$ abpo.2802

ISBN : 978-2-7535-3513-8

ISSN : 2108-6443

Éditeur

Presses universitaires de Rennes

Édition imprimée

Date de publication : 30 juin 2014

ISBN : 978-2-7535-3511-4

ISSN : 0399-0826

Référence électronique

Olivier Charles, "Le journal d'un chanoine du Mans. Nepveu de La Manouillère (1759-1807) ", Annales de Bretagne et des Pays de l'Ouest [En ligne], 121-2 | 2014, mis en ligne le 30 juin 2014, consulté le 23 septembre 2020. URL : http://journals.openedition.org/abpo/2802 ; DOI : https://doi.org/10.4000/ abpo. 2802 
XVII e siècle, si la place de Lyon joue un rôle dans les transactions financières avec les pays riverains de la Méditerranée, Amsterdam et Hambourg monopolisent l'activité bancaire en Europe du Nord. Savary conseille d'utiliser la ville hanséatique car la place Amsterdam est très liée au négoce hollandais qui cherche à nuire aux intérêts des marchands français. Outre ces questions, l'ouvrage traite tous les thèmes intéressant les professions mercantiles que ce soit la lettre de change, les compagnies privilégiées, les faillites, les courtiers, la comptabilité, les juridictions consulaires, le droit du travail...

L'ouvrage de Savary est plus qu'un pur manuel à destination des négociants, il décrit avec pittoresque la vie marchande de son temps et comme l'écrit avec justesse Henri Hauser, il y a dans ce texte " une poésie de la comptabilité, de l'épicerie, du commerce des étoffes. " La lecture du Parfait négociant est à recommander à toute personne s'intéressant au droit des affaires, à l'histoire du commerce mais aussi à l'histoire sociale. Sa présence est absolument obligatoire dans les rayons de toute bibliothèque de recherche digne de ce nom.

Pierrick POURCHASSE

Granger, Sylvie, Hubert, Benoît, TARoni, Martine, (ed.), Le journal d'un chanoine du Mans. Nepveu de La Manouillère (1759-1807), Rennes, PUR, coll. " Mémoire commune ", 2013, 738 p.

Trois chercheurs manceaux du Centre de Recherches Historiques de l'Ouest ont uni leurs compétences pour nous offrir en un épais volume très soigné la publication in extenso et la critique approfondie d'un important journal, un texte qui se trouve à la jonction de deux chantiers historiques aujourd'hui féconds : l'étude des écrits du for privé d'une part; celle des chanoines et du monde capitulaire d'autre part. Ce Journal d'un chanoine du Mans est d'autant plus précieux qu'il fait partie des rares journaux ou mémoires de chanoines séculiers qui nous soient parvenus. Il prend ainsi place aux côtés de ceux de Guillaume Baston, chanoine de Rouen jusqu'à la Révolution, et de quelques autres publiés à la fin du XIX ${ }^{\mathrm{e}}$ ou au début du Xx $\mathrm{x}^{\mathrm{e}}$ siècle (par exemple ceux de Jacques de Banne, chanoine de Viviers à la fin du Xvi et au début du XVII ${ }^{\mathrm{e}}$ siècle, de Pierre Le Folmarié, chanoine de Châlons dans la première moitié du XVII ${ }^{\mathrm{e}}$, de Mathieu Perrot, chanoine de Bourges à la fin du XVII , de Louis Le Gendre, chanoine de Notre-Dame de Paris au début du XVIII') et, surtout, de la précieuse édition critique du livre de raison tenu entre 1664 et 1684 par Jean-Gaspard de Grasse, chanoine de Cavaillon, due à Frédéric Meyer. Le Journal de René-Pierre Nepveu de La Manouillère est un écrit très riche dans lequel l'auteur, entre 1759 et 1807 , glisse progressivement du cérémonial au journal capitulaire, du journal familial à la chronique mondaine. La présente édition, scientifiquement irréprochable, est servie par un cahier d'illustrations très soigné - notamment les cartes et plans, très commodes pour les lecteurs les moins familiers des réalités mancelles - et par un imposant index des noms de personnes de 44 pages.

Le Journal du chanoine Nepveu est déjà bien connu des historiens du Maine en particulier et, plus largement, des spécialistes de l'histoire capitulaire. Cependant, le manuscrit original ayant depuis longtemps disparu, ils ne disposaient jusqu'à présent que d'une copie (1866-1867) et d'une édition ancienne (1877-1879) dues à l'abbé Gustave Esnault. Mais Sylvie Granger, Benoît Hubert et Martine Taroni nous démontrent que si la première est scrupuleuse et fiable, la seconde s'en écarte parfois malheureusement beaucoup. L'éditeur, cédant à de réels partis-pris idéologiques, 
religieux et sociaux, a en effet opéré de multiples coupes dans sa copie originale et procédé à " une réécriture discrète mais constante (p. 83) " du texte de Nepveu. Se fondant donc sur la copie initiale du texte et élargissant l'appareil critique au-delà de l'univers généalogique d'Esnault aux réflexions actuelles sur la culture des élites, les réseaux, le for privé et le monde capitulaire, les auteurs nous offrent assurément l'édition scientifique de référence du journal du chanoine Nepveu de La Manouillère.

L'intérêt majeur de ce texte est de mettre en scène un chanoine banal de l'ancienne France. À son contact, nous entrons en effet dans le petit monde aujourd'hui bien connu des chapitres cathédraux. Mais, ce qui ne nous était souvent que suggéré par les austères registres de délibérations, les longs statuts capitulaires et autres arides comptabilités s'anime soudain, et apparaît alors un portrait de chanoine ordinaire de l'Ancien Régime finissant. Nepveu est ainsi d'abord un chanoine issu des élites et parfaitement inséré dans la société urbaine, ce dont témoigne l'index de l'ouvrage qui recense pas moins de " 2000 personnages différents (p. 66) ». Né en 1732, après une enfance dorée, il fréquente la progéniture de l'élite mancelle au collège de l'Oratoire et au séminaire. Ordonné prêtre en 1756, il attend une prébende à la cathédrale, seul bénéfice jugé digne du statut familial. Il en prend possession en 1759 à 27 ans et sera l'homme d'un seul bénéfice. C'est alors qu'il entreprend la rédaction de son journal... dans lequel on découvre sans surprise une sorte de chronique mondaine ou de gazette locale. Il présente ainsi Le Mans et son diocèse au prisme des élites en une sorte de vaste base de données avant l'heure où de nombreuses précisions familiales côtoient des informations relatives à son tissu relationnel. À cet égard, le point d'orgue est incontestablement la réception donnée à l'occasion de la naissance du Dauphin en 1782 où soupent 70 personnes dûment nommées. Par ailleurs, Nepveu nous apprend qu'il fréquente des lieux révélateurs d'une certaine sociabilité des Lumières comme les sociétés de Concert et du Jardin de Saint-Vincent, une sorte d'académie.

Mais, comme tout bon chanoine, Nepveu est avant tout un spécialiste des affaires religieuses. Sous sa plume apparaissent, à l'occasion de cérémonies inusitées le plus souvent, les rites et usages en vigueur au sein du chapitre cathédral. Évocations de Te deum, de Fêtes-Dieu, des fêtes de l'Ascension, de l'exposition de la châsse de sainte Scholastique, de bénédictions de chapelles, des cérémonies du Jubilé... c'est un véritable cérémonial qu'il propose de fait à ses lecteurs. Il en est de même lorsqu'il décrit avec luxe détails les obsèques ou la prise de possession d'un évêque. À cette occasion, il montre que les affaires diocésaines retiennent aussi toute son attention. Dans le même registre, il nous dévoile le déroulement d'une période interépiscopale : entre janvier et juillet 1778 sont successivement évoqués le départ de l'évêque, la nomination de vicaires capitulaires, le siège vacant, l'arrivée et la prise de possession du nouvel évêque puis la mise en place d'une nouvelle administration épiscopale. Au-delà, son diocèse se limite le plus souvent aux nominations à la tête des cures dépendant du chapitre. En matière de vie capitulaire, il relève les prises de possession des chanoines et des membres du bas chœur puis en propose de rapides portraits.

Révélateur des réalités canoniales, Nepveu l'est encore par sa retenue. C'est en vain que l'on cherchera des jugements tranchés sur ses contemporains ou en matière théologique. De même, dès sa prise de possession, il se fond dans son chapitre et, en épousant les intérêts, fait corps avec lui. Dès lors, certains traits de sa personnalité s'effacent et il nous apparaît bien terne. Nepveu semble ainsi être, comme nombre de ses confrères à travers le royaume, un chanoine en définitive peu investi dans son chapitre. Il n'occupe pas d'office particulier au sein de sa compagnie, ne semble pas intéressé par l'accès à une quelconque dignité ou responsabilité. Ses préoccupations majeures sont la défense de la place du chapitre dans l'ordre 
social et, à l'intérieur de la compagnie, le maintien des hiérarchies. Son idéal? Celui du " très bon chanoine, bien exact à tous ses devoirs (p. 426) "... tout en restant, à l'occasion, sensible aux honneurs : dire la messe, porter les objets du culte pendant les processions...

Enfin, à partir de 1789, Nepveu apparaît comme un chanoine hostile au nouveau cours des choses mais manquant singulièrement de clairvoyance. Tout comme les chapitres à travers le royaume continuent à enregistrer le train-train capitulaire dans leurs registres, Nepveu consigne les événements habituels affectant sa compagnie - nomination d'un archidiacre, procession de la Fête-Dieu, décès d'un confrère... - et nourrit son carnet mondain - on ne compte plus les naissances, mariages ou décès. La pose des scellés à la cathédrale le 4 décembre 1790 marque certainement une rupture : dès lors, on sent une certaine irritation poindre. Après le serment de 1791, il refuse de croire "que les Cures de Ville qui, suivant le Décret, sont déclarées vacantes, soient remplies par ces mauvais sujets (p. 507) ", c'està-dire par les jureurs. Plus tard, il se laisse aller à un commentaire acerbe lors de l'élection de l'évêque constitutionnel du Mans. Puis, quand ce dernier ordonne ses premiers prêtres, il les qualifie de "rebut de l'ancien séminaire (p. 520) ". A-t-il pour autant compris que le royaume était entré dans une nouvelle ère? On en doute. Les États généraux ne valent que parce que " touttes les Compaignies y étoient, comme d'usage (p. 471) ", la prise de la Bastille et la Grande peur ne suscitent qu'un bref commentaire et un renvoi laconique " aux papiers publics de ce tems (p. 476)", l'abolition des privilèges le laisse de marbre (" On n'a jamais vu chanter un Te Deum pour si peu de chose ", p. 481). Plus tard, après bien des péripéties, il devient chanoine honoraire du chapitre concordataire mais " ce n'est plus comme autrefois (p. 613)" et il donne l'impression d'être un peu perdu dans un monde que n'est plus tout à fait le sien.

En définitive, nous tenons là un ouvrage qui est beaucoup plus que l'édition d'un écrit du for privé, un journal qui est beaucoup plus qu'un document local. Certes, à l'échelle mancelle, il permet de pallier l'absence des registres de délibérations du chapitre cathédral et dévoile de l'intérieur le fonctionnement de certains aspects de la complexe machinerie capitulaire. Mais, plus largement, il s'agit d'un travail de référence pour tous ceux qu'intéresse le monde des chanoines. L'imposante introduction scientifique (p. 13-87) non seulement dégage très clairement les centres d'intérêt du Journal mais constitue un véritable essai sur l'univers capitulaire, certes au prisme de l'expérience de Nepveu et du Mans... mais qui vaut pour bien d'autres chanoines et chapitres. Les références bibliographiques livrées en fin de volume ou en notes dans l'introduction permettent quant à elles de guider l'entrée parfois délicate dans le monde des chanoines. À cela, ajoutons le très complet glossaire de la vie canoniale (p. 657-679) appelé à devenir un instrument de travail essentiel et pratique puisque renvoyant systématiquement au texte de Nepveu.

Olivier CHARLES

RufFelet, Christophe-Michel, Les Annales briochines, 1771, Saint-Brieuc : histoire d'une ville et d'un diocèse, CHARLES, Olivier (éd. et dir.), Rennes/Saint-Brieuc, PUR/ Société d'émulation des Côtes-d'Armor, coll. " Mémoire commune ", Rennes, 2013, 625 p.

Né à Saint-Brieuc dans la bonne bourgeoisie cultivée de fonction et de commerce, Christophe-Michel Ruffelet fait ses études au collège de sa ville. Peuplée de 
6000 habitants, Saint-Brieuc est alors un siège épiscopal relativement médiocre, comme il y en a tant dans la France d'Ancien Régime, une cité qui se signale par une forte densité ecclésiastique. Ordonné prêtre en 1749, Ruffelet fréquente la boutique de Prud'homme, l'imprimeur et libraire briochin bien connu, et commence à rassembler sa belle bibliothèque, qui sera la plus importante de Bretagne, après celle du président Robien. Entré au chapitre collégial Saint-Guillaume en 1771, il n'accède au chapitre cathédral que trop tard (le 17 juillet 1789!); quelques mois plus tard, tous les chapitres de France sans exception sont éteints. Depuis longtemps, il aspirait à cette consécration; avec son cas, on a la preuve des nombreuses démarches et tractations pour accéder à un canonicat, qu'on ne fait généralement que subodorer. Emprisonné sous la Terreur, il récupère lors de son élargissement sa chère bibliothèque avec les fonctions de gardien de cette dernière. Cette collection de quelque 4000 ouvrages que l'inventaire révolutionnaire dressé dans l'été 1794 permet d'appréhender est au centre de sa vie, comme elle est au cœur de sa demeure. Elle est tout à la fois bibliothèque, cabinet de curiosité, cabinet scientifique. Logiquement assise sur une base religieuse, elle est cependant diversifiée et largement ouverte sur le monde contemporain. En 1803, Ruffelet réintègre le nouveau chapitre concordataire, mais meurt peu après, en 1806, léguant tous ses livres à l'évêque de Saint-Brieuc pour " être employés à l'instruction des séminaristes ". Ces milliers de volumes ont effectivement alimenté la bibliothèque de séminaire et la bibliothèque des Archives départementales.

La bibliothèque du chanoine avait été précocement le socle de ses investigations qui avaient abouti en 1771 à la publication à Saint-Brieuc de ses Annales, petit livre broché de quelque 250 pages, au prix abordable de 25 sols. Ces annales sont à vrai dire un curieux livre, constitué de plusieurs textes juxtaposés annonçant un ouvrage plus important qui ne verra jamais le jour. Après une description liminaire de son diocèse, Ruffelet se laisse porter par la chronologie, de l'Antiquité jusqu'en 1768. Il embrasse donc largement, des lointaines origines de la Bretagne jusqu'à " l'affaire de Bretagne " au service de laquelle il engage sa plume. Suivent dans l'esprit érudit de l'époque, des notices diverses, comme une liste documentée des paroisses du diocèse, la chronologie des évêques de Saint-Brieuc assortie de véritables biographies. La formule typée des annales égrène un bref récit année par année, les notices s'étoffant à l'époque moderne avec une information plus abondante et plus sûre. Pour le Moyen Âge, Ruffelet retient les épiscopats, les fondations religieuses, les faits d'armes ou bien des événements marquant pour l'histoire de la Bretagne comme cette famine de 1161 où les hommes furent "obligés à manger jusqu'à la terre " (p. 232). À l'époque moderne, son attention est plus spécialement retenue par les nombreuses fondations de la Réforme catholique et aussi les séquelles des guerres de Religion, comme le pillage de Saint-Brieuc en 1592 par l'armée des Espagnols et des Lorrains. Sur la longue durée pluriséculaire, le passionne l'histoire du duchépairie de Penthièvre dont " l'histoire est trop intéressante, trop intimement liée à l'histoire briochine " pour ne pas en faire un long tableau.

Ce seul texte en lui-même valait la publication et l'on peut remercier la Société d'émulation des Côtes-d'Armor d'avoir joué son vrai rôle de société savante en rendant un document de première importance facilement accessible aux lecteurs curieux ou érudits et à la communauté des chercheurs. Mais le présent ouvrage dépasse largement ce niveau, avec sa structure emboîtée. Non seulement y sont adjoints des notes, des commentaires, des analyses, des annexes le rendant parfaitement intelligible, mais encore, à un niveau supérieur, des études replaçant le tout dans l'historiographie contemporaine. L'ensemble est impressionnant par son ampleur, sa pertinence, son érudition qui est en prise avec les plus récents travaux. 
Pour mener à bien cette tâche ambitieuse, le maître d'œuvre, Olivier Charles, expert en études capitulaires, s'est adjoint une quinzaine de collaborateurs dont les compétences complémentaires " couvrent " la totalité de l'œuvre, jeunes chercheurs prometteurs et historiens confirmés. Les angles d'approche variés donnent du relief à l'ensemble. Impossible d'énumérer toutes les contributions : la moisson est impressionnante, allant bien au-delà de ce que l'on est en droit d'attendre pour la bonne intelligence d'un document. À titre d'exemple, on sait tout, ou presque, sur le Saint-Brieuc du chanoine Ruffelet, jusqu'au climat frais qui y régnait, grâce aux relevés météorologiques effectués entre 1772 et 1790 par Jean-Louis Bagot, docteur en médecine et maire de la ville. Parmi toutes ces contributions de qualité, la plus importante est sans doute celle d'Oliver Charles intitulée "Les chanoines historiens en France aux XVII et XVII e siècles ". Remarquablement informée et problématisée, c'est le point actuel sur cette question. Deux cartes suggestives y montrent que l'histoire capitulaire est le fait de la France septentrionale, Bretagne exceptée!

En historiens critiques aux bons sens du terme, les co-auteurs de cet ouvrage n'ont jamais pour Ruffelet la complaisance coupable, fréquente chez les biographes. Par exemple, pour son analyse de la société rurale, Yann Lagadec ose même dans son titre le terme fort des " œillères du chanoine Ruffelet ", lequel il est vrai se borne à des stéréotypes et ne voit guère les paysans. Curieusement, Ruffelet reste également muet sur les querelles religieuses sous l'Ancien Régime : rien sur le protestantisme, sur le jansénisme, sur les dévotions collectives... En fait, ces lacunes ne minimisent pas l'intérêt des Annales qui sont pour l'essentiel la construction d'une identité diocésaine. Dans ce domaine, Ruffelet n'est certes pas un pionnier; il s'inscrit dans une longue lignée de chanoines érudits comme Hiérosme Lopès à Bordeaux ou du célèbre abbé Lebeuf pour Auxerre puis Paris. Lui aussi veut honorer sa " patrie ", comme il le dit à plusieurs reprises, d'autant que son diocèse pâtit en Bretagne d'un déficit de notoriété. Ses annales sont porteuses d'un projet cohérent de "recharge identitaire ", prometteur aussi pour l'avenir du diocèse. Cela dit, Ruffelet n'innove en rien; il se conforme à la tradition pour laquelle l'histoire d'un diocèse demeure dans sa structure celle de ses évêques. Mais sur cette trame classique, les questions de fond ne sont pas éludées, comme les origines de la ville avec son saint éponyme. Ruffelet mentionne les indécisions autour de saint Brieuc ( $\mathrm{V}^{\mathrm{e}}$ ou $\mathrm{VI}^{\mathrm{e}}$ siècle?), il ne masque pas le "trou béant " entre saint Brieuc et le premier de ses successeurs à être attesté en 1032. C'est d'ailleurs, avec compétence qu'il se passionne pour l'histoire de l'Antiquité; plus de $10 \%$ des ouvrages sa bibliothèque ne relèvent-ils pas de cette période?

La France de l'Ouest s'est engagée résolument dans la publication de mémoires d'ecclésiastiques, comme Les Souvenirs d'un nonagénaire, en l'occurrence FrançoisYves Besnard, ou les Mémoires du chanoine Le Sage, toutes sources passionnantes. Certes, on ne saurait attendre des Annales Briochines des "tranches de vie ", comme on en trouve dans Le livre de raison de Jean-Gaspard de Grasse, chanoine de Cavaillon (éd. aux éditions du CTHS en 2002), ou le Journal du chanoine du Mans, René-Pierre Nepveu de La Manouillère (édité aux PUR en 2013); c'est simplement affaire de genre : les annales sont un exercice d'érudition, non propice aux faits divers souvent anodins ou aux épanchements.

Cet homme de la République des Lettres méritait de sortir de l'oubli, comme d'ailleurs l'ensemble de l'ordo canonicus longtemps négligé par la recherche historique, en fonction d'anachroniques jugements de valeur qui n'ont pas leur place en histoire. Le chanoine Ruffelet fut un polygraphe dont malheureusement sont perdus la plupart des écrits. Polygraphe, il l'était dans sa manière de travailler, honnête, méticuleuse, quasi mauriste; ses sources furent sans doute les archives de la collégiale Saint-Guillaume, sa bibliothèque et un réseau érudit difficile à cerner. Ses 
Annales briochines, tout à la fois riches et lacunaires, sont une source documentaire de premier plan, non seulement pour Saint-Brieuc, mais aussi pour la Bretagne et l'histoire en général, en particulier urbaine et religieuse.

Philippe LoupÈs

GOUBERT, Jean-Pierre, Malades et médecins à Saint-Malo à la veille de la Révolution, Rennes, PUR, coll. « Histoire ", 2013, 166 p.

Jean-Pierre Goubert considère " la première médicalisation " des années 17701780 en souhaitant mettre en valeur les originalités de la ville de Saint-Malo et de son " pays " le Clos-Poulet, la réponse d'une société face à la maladie et à la mort, ainsi que " le profond écart qui sépare la société locale de l'époque par rapport à notre début de XXI e siècle " (p. 11). Diverses sources, institutionnelles et médicales, sont mobilisées : archives hospitalières, dossiers sur les maladies épidémiques et les pauvres malades de la subdélégation de Saint-Malo, enquêtes sur le corps médical, les secours distribués ou topographies médicales locales. En revanche, la voix des malades demeure inaudible : livres de recettes, correspondances privées ou mémoires ne sont pas sollicités. En complément des documents d'archives, l'auteur s'appuie sur des études locales datant principalement du XIX ${ }^{\mathrm{e}}$ siècle et de la première moitié du $\mathrm{Xx}^{\mathrm{e}}$ siècle. De manière générale, les références historiographiques ne sont pas mentionnées, si ce n'est dans une bibliographie finale faisant une large place à l'historiographie française des années 1960-1980. L'impasse faite sur l'abondante bibliographie anglo-saxonne, venue notamment documenter depuis les années 1980 les modalités de la relation thérapeutique, nuit à la prise en compte des renouvellements historiographiques récents. La faible sollicitation des recherches francophones menées depuis les années 1990 empêche également Jean-Pierre Goubert de mettre en évidence les particularités malouines par rapport au cadre breton et national. Soulignons également que, tout en se défendant de moquer les représentations et pratiques du passé, l'auteur délivre une lecture positiviste, en opposant une pensée " scientifique " contemporaine (sans la définir pour autant) à des méthodes et travaux modernes " préscientifiques" (p. 15, 84, 104, 137).

Les deux premières parties de l'ouvrage, consacrées aux secours n'échappent pas à l'opposition entre " foi, croyances, coutumes " d'un côté (partie 1) et médecine " savante » de l'autre (partie 2), faisant fi des liens susceptibles d'unir ces pratiques : les pauvres malades rejoignant les institutions charitables ne sont-ils pas soignés aussi bien par les religieux que par les élites médicales de la ville? Les formations, pratiques de soins et rémunérations variées des soignants non " savants " (notables, religieux, dentistes et arracheurs de dents, matrones, oculistes, bandagistes, herniaires, rebouteux, renoueurs, remancheurs, rhabilleurs et autres guérisseurs) ne sont pas distinguées pour expliquer les choix thérapeutiques des malades. Les catégorisations sont rarement définies, à l'image des " charlatans " mêlant soignants empiriques, itinérants et même religieuses hospitalières! Au final, les classifications proposées semblent peu convaincantes et représentatives du point de vue des praticiens " savants " en lutte contre les autres recours, que l'auteur se défend pourtant d'adopter. En outre, ce dernier ne précise pas les raisons qui poussent les malades à choisir tel ou tel thérapeute, postulant que le premier recours est celui de la religion et des soignants " traditionnels ", la sollicitation des médecins et chirurgiens arrivant en second lieu, ce qui demeure contestable pour une frange non négligeable des malades de la période prérévolutionnaire. 